\title{
Left atrial volume: comparison of 2D and 3D transthoracic echocardiography with ECG-gated CT angiography.
}

\author{
Anish R Koka \\ Thomas Jefferson University \\ Stuart D Gould \\ Thomas Jefferson University \\ Alyson N Owen \\ Thomas Jefferson University \\ Ethan J. Halpern \\ Thomas Jefferson University
}

Follow this and additional works at: https://jdc.jefferson.edu/radiologyfp

Part of the Radiology Commons

Let us know how access to this document benefits you

\section{Recommended Citation}

Koka, Anish R; Gould, Stuart D; Owen, Alyson N; and Halpern, Ethan J., "Left atrial volume: comparison of 2D and 3D transthoracic echocardiography with ECG-gated CT angiography." (2012). Department of Radiology Faculty Papers. Paper 15.

https://jdc.jefferson.edu/radiologyfp/15

This Article is brought to you for free and open access by the Jefferson Digital Commons. The Jefferson Digital Commons is a service of Thomas Jefferson University's Center for Teaching and Learning (CTL). The Commons is a showcase for Jefferson books and journals, peer-reviewed scholarly publications, unique historical collections from the University archives, and teaching tools. The Jefferson Digital Commons allows researchers and interested readers anywhere in the world to learn about and keep up to date with Jefferson scholarship. This article has been accepted for inclusion in Department of Radiology Faculty Papers by an authorized administrator of the Jefferson Digital Commons. For more information, please contact: JeffersonDigitalCommons@jefferson.edu. 


\author{
As submitted to: \\ Academic Radiology \\ And later published as: \\ Left Atrial Volume: Comparison of 2D and 3D \\ Transthoracic Echocardiography with ECG-gated CT \\ Angiography \\ Volume 19, Issue 1, January 2012, Pages 62-68 \\ DOI: 10.1016/j.acra.2011.08.017
}




\begin{abstract}
Purpose: Left atrial volume (LAV) measurement by conventional 2-dimensional transthoracic echocardiography (2D-TTE) may be limited by the geometric model, by suboptimal definition of left atrial endocardium or by chamber foreshortening. 3D-TTE is posited to eliminate chamber foreshortening, and LAV by 3D-TTE should be more reflective of true LAV. The aim of our study was to compare conventional 2D-TTE and newer 3D-TTE measurements of LAV with MDCT measurements using automated chamber reconstruction (ACR).
\end{abstract}

Methods: Twenty two subjects consented to undergo 2D-TTE and 3D-TTE immediately prior to or following coronary CT angiography (cCTA) in this IRB-approved HIPAA-compliant study. LAV was calculated from 2D-TTE with the Area-Length method (ALM), and from 3D-TTE with ALM as well as with a 3D model (3DQ). ECG-gated cCTA was performed in helical mode. LAV was measured using ALM as well as ACR.

Results: LAV was significantly smaller by 2D-TTE $(80 \mathrm{ml} \pm 21)$ and 3D-TTE $(90 \mathrm{ml} \pm 24$ with ALM; 61ml \pm 16 with 3DQ) as compared with MDCT ACR $(120 \mathrm{ml} \pm 30), \mathrm{p}<0.01$. Correlation between MDCT ALM and MDCT ACR was excellent (mean delta $=-1.4 \pm 14 \mathrm{ml}, \mathrm{r}=0.91$ ). Correlation with MDCT ACR was no better for 3D-TTE $(r=0.80)$ than 2D-TTE $(r=0.80)$.

Conclusions: LAV is underestimated by both 2D-TTE and 3D-TTE relative to cCTA. Excellent agreement between ALM and ACR methods with MDCT suggests that the geometric model plays a negligible role in underestimation of LAV. Underestimation of LAV by echocardiography is likely related to suboptimal definition of left atrial contour. 


\section{Introduction}

Left atrial size is an important marker for a variety of clinical disease states. Elevated left atrial size predicts diastolic dysfunction ${ }^{1}$, severity of mitral regurgitation ${ }^{2}$, and the likelihood of success of pulmonary vein isolation in treating atrial fibrillation ${ }^{3}$. Increased left atrial size portends poorer outcomes in patients with atrial fibrillation ${ }^{4-6}$, myocardial infarction ${ }^{7-8}$ and dilated cardiomyopathy ${ }^{9}$. Thus, accurate measurement of left atrial size is of primary importance.

Historically, computed tomography (CT) and magnetic resonance imaging (MRI) have served as gold standards for left atrial volume, and 2 dimensional echocardiography (2D-TTE) was validated against these reference standards ${ }^{10-11}$. Echocardiography is currently the most common modality used to measure the left atrium because of its lower cost, portability and universal availability. The current recommended standard to estimate left atrial volume (LAV) as defined by the American Society of Echocardiography uses an ellipsoid geometrical model derived from two orthogonal apical views with the biplane area-length formula (ALM) ${ }^{12}$. Unfortunately, since the ALM depends on the acquisition of two non-foreshortened images, and depends on a geometric estimate, it is prone to errors.

Theoretically, 3 dimensional echocardiography (3D-TTE) should provide the most accurate evaluation of LA volumes as it is not prone to errors related to chamber foreshortening or geometric estimation. Until recently, however, 3D-TTE has had limited clinical applicability because of relatively laborious and time intensive acquisition and off-line processing ${ }^{13-14}$. The introduction of 'real time' 3D-TTE using matrix array transducers has resulted in sufficiently rapid image acquisition for clinical applications, but has not been rigorously validated ${ }^{15}$. 
Recently automated edge reconstruction algorithms on MDCT provides the ability to rapidly assess chamber volumes precisely and accurately ${ }^{16-17}$. The aim of this study was to use 3D-TTE software to measure the LAV and compare this to a CT reference standard to assess the accuracy of current generation 3D-TTE. 


\section{Methods}

\section{Patient Selection}

This HIPPA compliant study was approved by our local Institutional Review Board. From November 2009 to July 2010, consecutive subjects who were referred for cardiac MDCT during daytime hours (9AM-4PM) when a research sonographer was available were asked to participate in the study. MDCT studies and echocardiograms on any individual patient were performed consecutively with a minimum possible delay between the studies. All study subjects were 18 years or older and provided written informed consent prior to inclusion in the study. There were no exclusion criteria.

\section{Echocardiography}

Patients were positioned in the left lateral decubitus position. The echocardiogram was obtained either immediately before or after the cardiac MDCT. All measurements were performed off line using Xcelera, QLAB and 3DQ-Advanced software (Phillips Medical Systems, Andover, MA). 2D images were acquired using the S5-1 probe on a Phillips IE33 (Phillips Medical Systems, Andover, MA). Standard apical 4 and 2 chamber views were obtained. (Figure 1) The LAV was calculated using the biplane ALM as recommended by the ASE Guidelines. ${ }^{12}$ The formula applied was: A1 x A2 x 0.85/L, where A1 is the area in the apical 4 chamber view, A2 is the area in the apical 2 chamber view and L is the shorter length of the LA. These measurements 
were performed by two independent observers (AK and $\mathrm{AO}$ ) who were blinded to the MDCT results.

Apical real-time 3D images were acquired using the X3-1 matrix ultrasound probe on a Phillips IE33. (Figure 2) Apical full-volume images were acquired over 4 to 7 cycles. The image was aligned in order to obtain the best endocardial border definition prior to acquisition. The LAV was measured at end-systole as defined by the frame before opening of the mitral valve. The 3D longitudinal axes were aligned through the center of the left atrium to avoid foreshortening. Semi-automated border tracking was then performed by selecting four points around the mitral annulus (septal, lateral, anterior, inferior) and one point at the perceived apex, or superior border of the left atrium.. LA systolic volume was automatically calculated by the software using an edge detection algorithm. Endocardial borders were then checked and manually edited by two independent observers ( $\mathrm{AK}$ and $\mathrm{AO}$ ) who were blinded to the MDCT results.

In order to determine whether areas on 2D-TTE may be underestimated due to chamber foreshortening, we repeated the ALM measurements based upon areas obtained from nonforeshortened 4 and 2 chamber images from 3D-TTE.

Cardiac multi-detector computed tomography

All MDCT examinations were performed using a helical acquisition on a 256 slice iCT scanner (Philips Medical Systems, Cleveland, Ohio) during injection of 60-70 cc of iodinated contrast material (iodine concentration $350 \mathrm{mg} / \mathrm{ml}$ ). The standard scanning parameters for this study were set to $120 \mathrm{kVp}$ with $600 \mathrm{mAs}$ per section. Tube current was increased to $800-1000 \mathrm{mAs}$ per section on the basis of estimated weight for patients weighing 200-250 lbs (90.7-113.4 kg). 
ECG-based tube current modulation was used for dose reduction in all patients with regular heart rates at or below 65 beats per minute.

Transaxial images through the heart were reconstructed at $10 \%$ increments within the RR interval. Contiguous images were obtained with a slice thickness of $3 \mathrm{~mm}$. A cine loop encompassing the entire RR cycle was created with the 10 reconstructed phases, to identify the phase with the largest left atrial size. The systolic phase with the largest left atrial size was used to estimate left atrial volume with two different approaches. In order to calculate the left atrial volume using the standard echocardiographic approach, "echocardiographic views" were obtained by appropriately changing the plane and orientation of MDCT images to obtain four chamber and two chamber views. (Figure 3) Conventional 2D measurements of the left atrium were performed, and LAV was calculated using the ellipsoid model area-length method (ALM) based upon the American society of echocardiography chamber quantification guidelines. ${ }^{12}$ In order to measure left atrial volume with automated chamber reconstruction from the same data set, left atrial volume was quantified using post-processing software (EP Planning package on the Brilliance Workstation, Philips Medical Systems) with automatic edge detection of the left atrial endocardial borders: 3D automated chamber reconstruction (3D-ACR). (Figure 4). In order to best match the volume measured by echocardiography, the pulmonary veins and left atrial appendage were manually excluded from 3D-ACR measurements. Two independent observers blinded to the TTE results (AK and $\mathrm{EH}$ ) independently performed all measurements on MDCT.

Statistical analyses 
Statistical Analysis was performed with SAS software version 9.1. Data are expressed as means \pm standard deviation unless otherwise indicated. Initial interpretations of 2D-TTE, 3D-TTE and MDCT were performed by two independent readers; These two readings were used to compute intramodality agreement. Intermodality correlation was assessed between the most experienced reader in each modality. The correlation between any 2 methods was determined using Pearson's correlation. The Bland-Altman method was used to measure the limits of agreement between 2 methods. Interobserver variability was determined for 2D-TTE, 3D-TTE and MDCT using the coefficient of variability calculated as the mean of the absolute differences between 2 measurements divided by the average of the 2 measurements, expressed as a percentage. 


\section{Results}

Twenty three subjects were consented to participate in the study. One patient was excluded because the presence of a mitral prosthetic mechanical valve precluded accurate visualization of the endocardial borders of the left atrium. All echocardiograms were performed either immediately before or after cardiac MDCT image acquisition such that both studies were completed within one hour. Pertinent clinical information is detailed in Table 1.

Left atrial volumes were noted to be significantly smaller by $2 \mathrm{D}(80 \mathrm{ml} \pm 21)$ and 3D TTE $(61$ $\mathrm{ml} \pm 16)$ than MDCT ACR $(120 \mathrm{ml} \pm 30)$ with $\mathrm{p}<0.001$ for both comparisons. The currently accepted 2D-TTE standard (ALM) fared well with good correlation but with large limits of agreement as compared to MDCT ACR $(r=0.80, \Delta=-38 \pm 20 \mathrm{ml} \mathrm{p}<.0001)$. Using the semiautomated border tracking available to us on the Phillips system , we measured 3D-TTE LAV's, manually edited them, and compared them to MDCT ACR. These 3D-TTE semi automated volumes (3D-TTE SAV) showed good correlation to MDCT ACR, similar to 2D-TTE with an $\mathrm{r}=0.80$, but with a greater bias than $2 \mathrm{D}-\mathrm{TTE}$ with a $\Delta=-58 \pm 20 \mathrm{ml}, \mathrm{p}<.0001$. The unedited 3DTTE LAVs had poorer correlation with MDCT ACR $(r=0.69)$ with wide limits of agreement $(\Delta$ $=-76 \pm 23 \mathrm{ml})$. Means, correlations, bias and limits of agreement for 2D-TTE, 3D-TTE and MDCT ALM are listed in Table 2.

In order to better understand the sources of error and underestimation on echocardiography, we used MDCT to generate orthogonal apical 4 and 2 chamber views, and used the same ALM on these images to derive a LAV. Correlation between MDCT ALM and MDCT ACR was excellent, with a very small bias and narrow limits of agreement $(\Delta=-1.4 \pm 14 \mathrm{ml}, \mathrm{r}=0.91, \mathrm{p}=$ 0.69), suggesting that the geometric model is not responsible for most of the difference between 
2D and 3D methods. In order to determine whether foreshortening of the left atrial chamber on 2D-TTE was responsible for the lack of agreement with MDCT, ALM computations were also performed based upon 3D-TTE acquisitions of 2 and 4 chamber views. Using the ALM on the 3D-TTE images resulted in larger volume and thus a smaller bias $(\Delta=-28 \pm 22 \mathrm{ml}, \mathrm{p}<.0001)$, but with no improvement in correlation to MDCT ACR ( $(\mathrm{r}=0.80)$ and with persistently wide limits of agreement.

Interobserver agreement was noted to be best for the MDCT ACR reference standard, with a mean interobserver variability of only 4\%. 2D-TTE, 3D-TTE and MDCT ALM all had similar interobserver variabilities (9-11\%). (Table 3) 


\section{Discussion}

Although left atrial size and LAV are quantified in the standard echocardiography examination, these measurements are limited by operator dependence of the examination which may result in chamber foreshortening, as well as by imprecision related to geometric assumptions in the standard biplane ALM computation. Real time 3D-TTE has promise to improve the quantitative nature of echocardiography in clinical practice because of its ability to capture a full volume of data which result in the acquisition of non-foreshortened images. We tested this hypothesis in this study by comparing 3 dimensional echocardiography and the currently accepted standard on 2 dimensional echocardiography (ALM) to a MDCT reference standard. We show that 3D-TTE correlates well with MDCT but is no better than 2D-TTE with respect to correlation, bias, limits of agreement or interobserver variability.

In order to better understand the sources of error for echocardiographic measurements of the left atrium we applied the same ALM on MDCT to estimate left atrial volume. We show excellent correlation with minimal bias between MDCT ALM and MDCT ACR, suggesting that the geometric estimate plays a very small role in the error we see with echocardiography. The error appears to be due to the smaller apical 4 and 2 chamber areas measured on 2D-TTE compared to MDCT. In order to understand whether the smaller areas on 2D-TTE are related to poor endocardial definition or to chamber foreshortening by 2D-TTE, we measured the areas on nonforeshortened 4 and 2 chamber images from 3D-TTE. While volumes obtained from nonforeshortened 3D-TTE images were slightly larger than their 2D-TTE counterparts, correlation with MDCT ACR was no better for 3D-TTE than 2D-TTE $(r=0.80)$. We conclude that while chamber foreshortening may result in some underestimation of LAV by 2D-TTE, it does not 
explain the lack of excellent correlation between echocardiography and MDCT. A major limitation to accurate left atrial volumes by echocardiography is likely related to suboptimal definition of the left atrial contours. Suboptimal definition of these contours may be related to poor resolution in the far field. As 3D-TTE generally has lower resolution than 2D-TTE, correlation with MDCT is not improved by 3D-TTE.

Three dimensional echocardiography has been used to measure left atrial volumes with mixed success in the past. Rodevand et. al. examined LAV by both 2D-TTE and 3D-TTE compared to an MRI reference standard. ${ }^{13}$ Despite a laborious 3D image acquisition that entailed the simultaneous collaboration of two investigators, the authors found 3D-TTE was no better than 2D-TTE in measuring LAV. Keller et. al., used a freehand scanning technique which relied on manually tracing multiple cross-sectional areas through the left atrium and combining them in a polyhedral surface reconstruction algorithm to generate a volume. ${ }^{14}$ In this study 3D-TTE demonstrated the best correlation $(\mathrm{r}=0.91)$ with the smallest bias and narrowest limits of agreement $(5 \pm 22 \mathrm{ml})$, but this was only marginally better than $2 \mathrm{D}-\mathrm{TTE}(\mathrm{r}=0.87,14.4 \pm 24 \mathrm{ml})$. Both studies also utilized methods that are time and labor intensive and not practical for use in a busy clinical lab.

The recent development of 3D matrix array transducers which can rapidly acquire a volume of data in real time, and the simultaneous development of post-processing software with semiautomated edge detection promises to move 3D quantification from the research lab to the clinical lab. Only one study to date, however, has attempted to validate the 3D volumes acquired in this manner to a reference standard. Artang et. al., found significant correlation using 3D-TTE for left atrial systolic volumes ( $\mathrm{r}=0.86)$ when compared to an MRI reference standard with better 
interobserver variability (5 vs. $12 \%$ ), but do not present their data comparing 2D-TTE left atrial

systolic volumes to MRI. ${ }^{15}$ Impressively, they noted the semi-automated volumes demonstrated good correlation that was not improved upon with manual editing. In this study, we found correlation using 3D-TTE was not significantly better and did not result in less bias or narrower limits of agreement. Our findings differ from Artang in two important respects: the semiautomated volumes that were not manually edited demonstrated poor correlation, and our interobserver variability was no better than 2D-TTE. Importantly, this suggests that while current generation real time 3D-TTE is no worse than 2D-TTE for quantification, it is not ready to displace 2D-TTE in the clinical setting for LAV measurement.

\section{Limitations}

This study is limited by its small sample size which may over-emphasize the impact of an individual data point on the results. However, our results for the 2D-TTE ALM and MDCT measurements are consistent with prior studies ${ }^{16}$. Another limitation is that the real time $3 \mathrm{D}-$ TTE measurements rely on an algorithm designed to model the left ventricle. While this method did demonstrate correlation, it is possible that correlation could be improved with improvements to this software focused on modeling the complex contour of the in-vivo left atrium. Lastly, our reference standard uses MDCT which has a lower temporal resolution than echocardiography. The lower temporal resolution of MDCT may result in underestimation of LAV when imaging is not obtained at the time of maximum atrial filling, Correlation between MDCT and TTE may be worse than expected because the left atrium is measured at a slightly different point during the RR cycle on CT and on echocardiography. Nonetheless, the lower temporal resolution of MDCT 
may result in a smaller LAV, but cannot explain the substantially larger LAV measured with MDCT as compared with TTE.

\section{Conclusion:}

Imaging the left atrium more precisely and accurately may allow clinicians to better predict success after pulmonary vein isolation, the risk of stroke in patients with atrial fibrillation, or help quantify the severity of mitral regurgitation. 2D-TTE is often the first imaging study used for evaluation of cardiac morphology, as echocardiography is widely available, non-invasive, relatively inexpensive, not limited by arrhythmias and does not involve ionizing radiation. Real time 3D-TTE has been well validated and has been shown to be superior to 2D-TTE for quantifying volumes and mass of the left ventricle (12), but we do not find the same true when imaging the left atrium. While real time 3D-TTE correlates well with our reference standard, it proves to be no better than 2D-TTE, likely due to poor definition of atrial borders in the far field. Improving correlation with current generation real time 3D-TTE may not be feasible by imaging the left atrium from the apical views. Efforts to improve the assessment of left atrial volume by echocardiography should concentrate on better techniques for defining the endocardium that outlines the left atrial chamber. Indeed, the only study to date that demonstrated excellent correlation with minimal bias imaged the left atrium from the parasternal window ${ }^{14}$, thus placing the left atrium much closer to the ultrasound transducer. Future studies exploring the feasibility of this approach with real-time 3D-TTE are needed. 
Figure Captions

Figure 1. Standard transthoracic echocardiographic views with measurements of left atrial area used for calculation of left atrial volume. (A) Apical 4 chamber view. (B) Apical 2 chamber view.

Figure 2. Three dimensional acquisition of the left atrium using 3DQ-Advanced software (Phillips Medical Systems). Images are acquired as a single volume, and a model of the left atrium is automatically created to compute left atrial volume.

Figure 3. MDCT acquisition of the heart presented in standard 'echocardiographic' views. The left atrium is outlined in black, excluding the pulmonary veins. These views are used to calculate left atrial volume with based upon the ALM. (A) Apical four chamber view. (B) Apical two chamber view.

Figure 4. Automated chamber reconstruction (EP Planning package, Brilliance Workstation, Philips Medical Systems) with MDCT data. (A) The left atrium with associated pulmonary veins and left atrial appendate is detected. (B) The pulmonary veins are manually cropped out. (C) The left atrial appendage is manually cropped out to create the model of the left atrium used for determination of LAV. 


\section{References}

1. Nagueh S et. al., Recommendations for the Evaluation of Left Ventricular Diastolic Function by Echocardiography. J Am Soc Echocardiogr. 2009;22(2): 107-33.

2. Zoghbi W et. al., Recommendations for Evaluation of the Severity of Native Valvular Regurgitation with Two-dimensional and Doppler Echocardiography. J Am Soc Echocardiogr 2003;16:777-802.

3. Montefusco A, Biasco L, Blandino A, Cristoforetti Y, Scaglione M, Caponi D, Di Donna P, Boffano C, Cesarani F, Coin D, Perversi J, Gaita F. Left atrial volume at MRI is the main determinant of outcome after pulmonary vein isolation plus linear lesion ablation for paroxysmalpersistent atrial fibrillation. J Cardiovasc Med (Hagerstown). 2010 Aug;11(8):593-8.

4. Benjamin EJ, D’Agostino RB, Belanger AJ, Wolf PA, Levy D. Left atrial size and the risk of stroke and death: the Framingham heart study. Circulation 1995;92:835-41.

5. Bolca O, Akdemir O, Eren M, Dagdeviren B, Yildirim A, Tezel T. Left atrial maximum volume is a recurrence predictor in lone atrial fibrillation: an acoustic quantification study. Jpn Heart $\mathbf{J}$ 2002;43:241-8.

6. Di Tullio MR, Sacco RL, Sciacca RR, Homma S. Left atrial size and the risk of ischemic stroke in an ethnically mixed population. Stroke 1999;30:2019-24.

7. Moller JE, Hillis GS, Oh JK, Seward JB, Reeder GS, Wright RS, et al. Left atrial volume: a powerful predictor of survival after acute myocardial infarction. Circulation 2003;107:2207-12.

8. Beinart R, Boyko V, Schwammenthal E, Kuperstein R, Sagie A, Hod H, et al. Long-term prognostic significance of left atrial volume in acute myocardial infarction. J Am Coll Cardiol 2004;44:327-34.

9. Modena MG, Muia N, Sgura FA, Molinari R, Castella A, Rossi R. Left atrial size is the major predictor of cardiac death and overall clinical outcome in patients with dilated cardiomyopathy: a long-term follow-up study. Clin Cardiol 1997; 20:553-60.

10. Kircher B et. Al., Left atrial volume determination by biplane two-dimensional echocardiograpy: Validation by cine computed tomography. Am Heart J. 1991 Mar;121:864-71.

11. Jarvinen V et. Al., Assessment of Left atrial volumes and phasic function using cine magnetic resonance imaging in normal subjects. Am Jrnl Card. 1994;73:1135-38.

12. Lang RM, Bierig M, Devereux RB, Flachskampf FA, Foster E, Pellikka PA, Picard MH, Roman MJ, Seward J, Shanewise JS, Solomon SD, Spencer KT, Sutton MS, Stewart WJ; Chamber Quantification Writing Group; American Society of Echocardiography's Guidelines and 
Standards Committee; European Association of Echocardiography. Recommendations for chamber quantification: a report from the American Society of Echocardiography's Guidelines and Standards Committee and the Chamber Quantification Writing Group, developed in conjunction with the European Association of Echocardiography, a branch of the European Society of Cardiology. J Am Soc Echocardiogr. 2005 Dec;18(12):1440-63.

13. Rodevand $\mathrm{O}$ et. Al., Left atrial volumes assessed by three and two-dimensional echocardiography compared to MRI estimates. Int Jrnl Card Imag. 15: 397-410, 1999.

14. Keller AM, Gopal AS, King DL. Left and right atrial volume by freehand three-dimensional echocardiography: in vivo validation using magnetic resonance imaging. Eur J Echocardiogr. 2000 Mar;1(1):55-65.

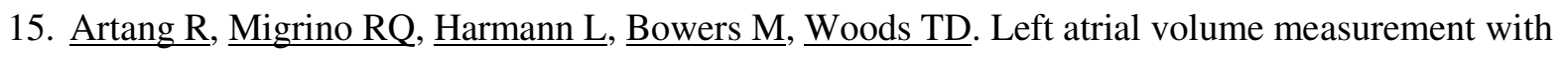
automated border detection by 3-dimensional echocardiography: comparison with Magnetic Resonance Imaging. Cardiovasc Ultrasound. 2009 Mar 31;7:16.

16. Koka AR, Yau J, Van Why C, Cohen IS, Halpern EJ. Underestimation of left atrial size measured with transthoracic echocardiography compared with 3D MDCT. AJR Am J Roentgenol. 2010 May;194(5):W375-81.

17. Christiaens L, Lequeux B, Ardilouze P, Ragot S, Mergy J, Herpin D, Bonnet B, Allal J. A new method for measurement of left atrial volumes using 64-slice spiral computed tomography: comparison with two-dimensional echocardiographic techniques. Int J Cardiol. 2009 Jan 9;131(2):217-24. Epub 2008 Jan 4. 